\title{
A Research on Interbank Loan Interest Rate Fluctuation Characteristics and the VaR Risk of China's Commercial Banks
}

\author{
Baoqian Wang, Cheng Wang, Xikun Zhang \\ Business School, Hohai University, Nanjing, China \\ Email: Bq64@163.com, hhucwangcheng@126.com, zxk19870910@163.com
}

Received June 20, 2012; revised July 22, 2012; accepted August 1, 2012

\begin{abstract}
According to the historical time series data of commercial interbank, this paper examines the interest rate fluctuation distribution characteristics, indicating that EGARCH Model can better fit the rate volatility of the interbank market interest. This paper calculates the value at risk (VaR) of five major commercial banks using EGARCH Model with such a conclusion that the difference that major commercial banks face is various. The interest risk of state-owned commercial banks and other financial institutions is more serious than the city commercial banks and foreign banks. The interest risk of rural credit cooperatives is the least serious.
\end{abstract}

Keywords: GARCH Model; Interest Rate; VaR

\section{Introduction}

Since 1996, the speed of China's market interest rate process put fast gradually, letting go the interest rate of the inter-bank market interest treasury bonds and policy financial bonds. Domestic and foreign currency loans and large foreign currency deposit rates are also involved. Long-term RMB large deposit agreement is available and at the same time china gradually expands the floating range of RMB deposit and lending rates. With the market operation of china's interest rate, the issue of interest rate risk measurement and management has become unavoidable. By using statistical knowledge and VaR model, this paper analyzes China's interest rate market risk distribution patterns and quantifies the interest rate risk of commercial banks in China.

\section{Literature Review}

Since 1978, china's commercial banks have experienced a series of management system reforms. But before 1993, commercial bank interest rate has always been under control, never out of the planned economic system. In this period, domestic focus is on the research of the regulation of commercial bank interest rate. Huang Jianfeng (2001) systematically expounds on the normative operations of the commercial bank deposit and loan interest rate [1]. Ge Qi (2003) systematically introduces foreign measurements and management methods on interest rates. In practice, he actively explores the advanced technologies and methods to be used in commercial banks' interest rate risk management approach [2]. Dai Guoqiang (2005) uses the VaR method, the stochastic analysis method and the option pricing method to put forward the choice of benchmark interest rate of china's commercial banks, together with the interest rate trend forecast in order to effectively control the interest rate risk, and enhance their own competitiveness [3].

China's interbank market interest rate research begins relatively late. In the early days; it is primarily based on qualitative analysis, but in recent years it terms into quantitative analysis. Tang Qiming and Gao Xiang (2002): interbank market interest rate of different terms is in line with the expectations of interest rate term structure theory [4]. Xu Hanfei (2004): by using ECM model, $\mathrm{Xu}$ studies the transmission mechanism of central bank loan interest rate to interbank loan interest rate transmission, and finds that the transmission mechanism is asymmetric and dynamic, and the adjustment that central bank transmits to refinancing interest rate is only about $60 \%$ direct conducted to the inter-bank market interest rates [5]. Lin Hai, Zheng Zhenlong (2003): by integrating Vasicek model and CIR model into GARCH model, Lin and Zhang discusses china's interest rates, but they don't give a further research on rate volatility [6]. Dong Le (2006): Dong validates the dual asymmetry of china's short-term interest rate in terms of mean velocity and the conditional variance. He gives the features that the correlation coefficient of the inter-bank market 
interest buyback rates is close to 1a suppose, namely, foreign classic model doesn't create very effective results about the prediction of the term structure of interest rate, which will even worse than the random walk. But he has no further validation [7]. Zhang Na, Huang Xinfei, Liu Deng (2006): in the process of constructing GARCH model to analysis China's various term interbank market interest rate volatility, they discover GARCH model can better explain the interbank market interest rate fluctuation. Such fluctuation and the lag period fluctuation are smooth and show a smoothly decreasing trend, but the volatility is not serious [8]. Zheng Yaotian, Du Ziping (2007): Zheng and Du fit the inter-bank overnight rates by using EGARCH model, finding out the EGARCH (1, 3) model the best fitting one, which fully embodies the overnight lending market information asymmetry. They also do the short term prediction by EGARCH $(1,3)$ and obtain ideal forecasting effect, thereby determine a suitable model for China's interbank market interest rate forecasting [9]. Li Jie, Gao Ning, Chai Jun (2007): Li and Gao establish AR (P)-EGARCH model in China's interbank market. From liquidity, transaction volume and structural changes terms, they explore the various influence factors for daily overnight interest rates of varieties, proving that the positive leverage effect exists for the sequence fluctuation in overnight lending market [10]. Liu Xiangyun (2007): doing a theoretical and practical analysis for china's commercial bank interest rate risk in terms of interest rate term structure on the basis of drift-jumping methods. A mean reversion phenomenon is discovered in the short term market interest rate, such as 7-day period Chinese bond repurchase rate and 7 days interbank rates [11].

The VaR method was first adopted in J. P. Morgan risk management practice of the last 90th century. Philippe Jore (1995) publishes the master piece systematically introducing VaR and its application. Philippe Jore dose the all round discussion about VaR's mathematical statistics basis, calculation process and ways of application [12].

Chew \& Lilian (1996): summing up the calculation parameter method for $\mathrm{VaR}$, historical simulation method and the Moncaro simulation method [13]. Futher more, Duffle \& Pan (1997) and Jorion (2000) respectively does profound summary in Risk Value and Value at Risk: Risk Control Reference. Among them, the application of various methods to compare the effects of the research is particularly enlightening $[14,15]$.

To sum up, foreign researches mainly focus on the model application, while the domestic researches mainly concentrate on the model selection, rarely on risk quantitative analysis and forecast. China's interbank offered rate (CHIBOR) is China's first market-oriented interest rates, so it could sensitively react to the supply and de- mand situation in monetary market. As a result, the interbank interest rate can be regarded as the benchmark interest rate for China's monetary market. With this hypothesis, the paper tests the applicability of GARCH model and makes a short-term prediction for the 7 days' interest rate. Given a certain degree of confidence of china's commercial banks, based on the fitted model and various periods of different variance, by using bank interbank borrowing trading positions, the commercial bank risk values can be measured and compared analysis will be available.

\section{The Selection and Construction of Interest Rates Model}

\subsection{The Normality Test for the Sample of Inter-Bank Interest Rate}

This paper uses the interbank market interest rate as the market interest rate (Shanghai Interbank Offered Rate, referred to as Shibor )to inspect china's commercial bank lending position risk. The purpose is to reflect the interest risk of China's commercial banks under the background of market orientated interest. At present, been announced Shibor varieties includes overnight, 1-week, 2-week, 1-month, 3-month, 6-month, 9-month and 1-year. This paper analyzes the data from 2007 to 2011, according to the 1-week Shibor.

Sample of this paper is from 01/01/2007 to 31/12/2011. There is totally 1249 statistics of 1 -week Shibor. And the fitting prediction sample is from the first quarter of 2012. Data processed by Eviews software.

From 1-week Shibor wave pattern (Figure 1), it could be seen that the market interest rate violates strongly, so the sequence is not stable. In order to get a stable return time series data, some techniques need to be done. Using the equation $y_{t}=\log r_{t}-\log r_{t-1}$ for the logarithm of a first-order differential treatment, and make its become more stable income sequence (Figure 2).

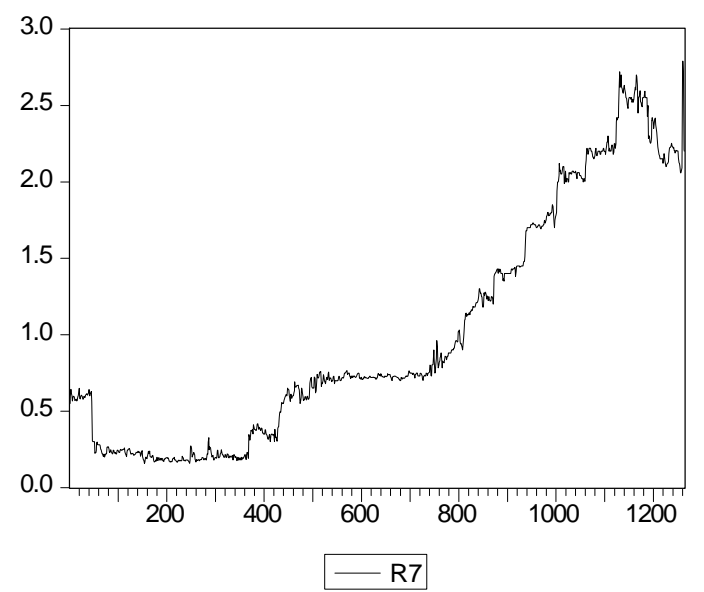

Figure 1. 1-week shibor rate fluctuation. 


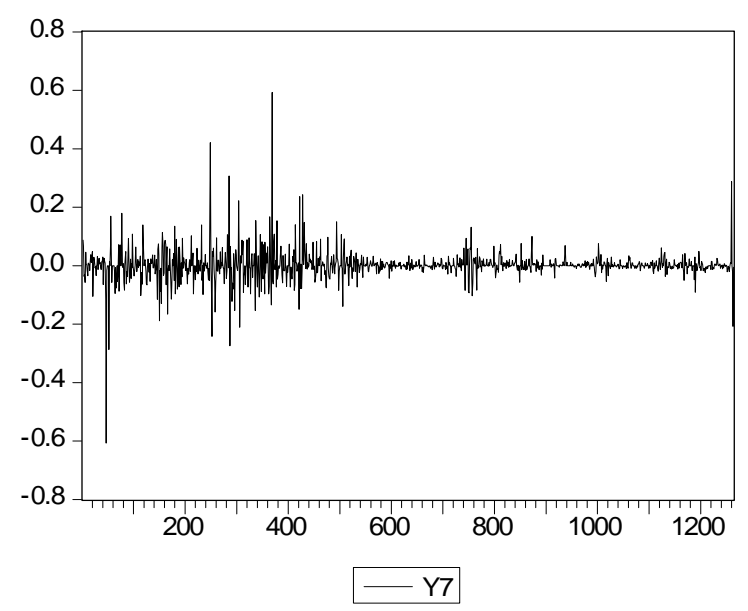

Figure 2. The rate difference fluctuation figure. Data sources of Figures 1 and 2: http//:www.bba.org.uk/; http//:www.chinamoney.com.cn/.

$r_{t}$ : Time series of daily interbank interest rate market.

Do the normality test using the differential logarithmic statistics and the results (Table 1) show that, in the 1-week inter-bank lending market, the average rate of return is 0.001097; the skewness (S) is 0.419660; the kurtosis $(\mathrm{K})$ is 42.33005; the statistic value of J-B test of normality is 81398.90 with a probability of 0 . Information got from the above statistical value is that the volatility of the return rate is strong and the time series is not in line with the normal distribution for the presentation of "fat-tail phenomenon". The intermediate portion contains a large amount of statistical information. Therefore, we need to use the GARCH model distribution instead of normal distribution so as to improve the model fitting effects.

\subsection{Inspection of ARCH Effects}

Because of the different availability of information in different moments, financial time series usually has a peak, thick tail and cluster characteristic. In order to deal with these characteristic, Engle proposed "Conditional Heteroscedasticity Regression Model” (Auto Regressive Conditional Heteroscedasticity, ARCH Model) to deal with such problems. The model uses a regression form and all the available information to describe the variance of the variance. In 1986, Bollerslev proposed "GARCH Model” (Generalized Auto Regressive Conditional Heteroscedasticity Model), which was also called the generalized autoregressive conditional heteroscedasticity model. It is the expansion from of the most basic conditional variance function ARCH model, and it has a strong advantage in the explanation and establishment of variance of time series. In 1990, Zakoian proposed "TARCH Model” (Threshold ARCH), namely the asymmetric conditional heteroscedasticity regression model. It explains the phenomenon in the stock market that while the crash and the roar margin are the same, the crash process is always accompanied by the serious fluctuation. In 1991, Nelson proposed "EGARCH Model”, it effectively depict the conditional variance of positive, negative interference of the asymmetric response.

The above analysis shows that china's commercial bank lending rate distribution does not conform to the normal distribution hypothesis, therefore, through a variety of conditions heteroscedastic model comparison, we need select the optimal GARCH model to fit china's commercial bank interbank market interest rates.

\subsubsection{Autocorrelation Test}

Do the autocorrelation test on Y7 on a delay of 18 orders, and the test results are shown in Table 2. On the 0.05 significance level, the probability $\mathrm{P}$ value was 0 , which is less than 0.05. Inspection instructions show that until higher-order sequence, the yields still remain a strong correlation relationship. So now the return series autoregressive model is being fitted. Given the yield of the sequence $\left\{y_{t}\right\}$ after autocorrelation and partial autocorrelation test, the following model is selected to fit the sequence $\left\{y_{t}\right\}$. Use maximum likelihood estimation method to estimate the parameters.

$$
y_{t}=c+\phi_{1} y_{t-1}+\phi_{2} y_{t-2}+\phi_{3} y_{t-3}+\varepsilon_{t}
$$

\subsubsection{The Stationary Test}

Use the unit root test (Table 3) to check the smooth of sequence. As the stationary of the sequence directly affect the model fitting result, and the non-stationary data sequence will produce a false return we have to test the stationary of the sequence. Here we use the method of Augmented Dickey-Fuller (ADF) to make unit root test for yield sequence $\left\{y_{t}\right\}$. The ADF value is -17.20466 , which is less than $1 \%$ and $5 \%$ significance level. So the unit root

Table 1. Normal inspection result.

\begin{tabular}{lcccccc}
\hline Mean & $\begin{array}{c}\text { Standard } \\
\text { deviation }\end{array}$ & $\begin{array}{c}\text { Partial } \\
\text { degree }\end{array}$ & Kurtosis & $\begin{array}{c}\text { J-B } \\
\text { statistics }\end{array}$ & P value \\
\hline Y7 & 0.002 & 0.050760 & 0.419550 & 42.32008 & 81398.90 & 0.000000 \\
\hline
\end{tabular}

Data sources: The result of the empirical study by using the software of Eviews. Statictics database: Figures 1 and 2 http//:www.bba.org.uk/; http//: www.chinamoney.com.cn/.

Table 2. The autocorrelation test of yields.

\begin{tabular}{ccc}
\hline & 7 days lending of inter-bank \\
\hline Delay in order & Q-statistic & P value \\
$\mathbf{6}$ & 56.492 & 0.000 \\
$\mathbf{1 2}$ & 71.981 & 0.000 \\
$\mathbf{1 8}$ & 77.592 & 0.000 \\
\hline
\end{tabular}

Data sources: The result of the empirical study by using the software of Eviews. Statictics database: Figures 1 and 2 http//:www.bba.org.uk/; http//: www.chinamoney.com.cn/. 
hypothesis are rejected by the test values, indicating that the sequence of yield $\left\{y_{t}\right\}$ is a stationary sequence.

\subsubsection{ARCH Test}

In order to see whether the Conditional Heteroscedastic exists in the return series $\left\{y_{t}\right\}$, the paper uses Lagrange multiplier method to do the test. Make a 3 lag ARCH effect test for the residuals of the regression equation, and from the test results (Table 4), we could see that the F-statistic and the LM-statistic probability value is relatively 0.000227 and 0.000241 , less than 0.05 . The original assumptions rejected hence ARCH effect exists in the model.

\subsection{Interest Rate Model Selection}

Compare ARCH, GARCH, EGARCH and TARCH model in terms of the residuals, depending on the Akaike Information Criterion (AIC), Schwarz (SC) guidelines and the LL principle of maximum likelihood function value, and hence select the most suitable model.

The modeling process: select the inter-bank lending of 1249 continuous-time series data and compare the residuals of ARCH (1), GARCH $(1,1)$, TGARCH $(1,1)$ and EGERCH $(1,1)$ (Appendix 1). The appendix shows the AIC and SC of EGARCH $(2,1)$ is the smallest; the LL value of TGARCH $(1,1)$ is smaller than EGARCH (2, $2)$; the fitting coefficients of $\operatorname{EGARCH}(2,1)$ are higher than TGARCH $(1,1)$. Consider the overall results; EGARCH $(2,1)$ model is selected to fit the inter-bank interbank offered rate sequence

According to the results of fitting model, the 7-day inter-bank lending yield model is:

$$
y_{t}=-0.117429 y_{t-2}+\varepsilon_{t}
$$

Table 3. The unit root test results.

\begin{tabular}{llll}
\hline & ADF statistics & \multicolumn{2}{l}{ Critical value } \\
\hline & & $1 \%$ & -3.4384 \\
$\begin{array}{l}\text { Inter-bank 7 } \\
\text { day lending }\end{array}$ & -17.20466 & $5 \%$ & -2.8643 \\
& & $10 \%$ & -2.5682 \\
\hline
\end{tabular}

Data sources: The result of the empirical study by using the software of Eviews. Statictics database: Figures 1 and 2 http//:www.bba.org.uk/; http//: www.chinamoney.com.cn/.

Table 4. ARCH effect test.

\begin{tabular}{ccccc}
\hline \multicolumn{5}{c}{ ARCH Test } \\
\hline $\begin{array}{c}\text { Inter-bank 7 } \\
\text { day lending }\end{array}$ & F-statistics & 4.820709 & $\mathbf{P}$ & 0.000227 \\
& LM-statistics & 23.76079 & $\mathbf{P}$ & 0.000241 \\
\hline
\end{tabular}

Data sources: The result of the empirical study by using the software of Eviews. Statictics database: Figures 1 and 2 http//: www.bba.org.uk/; http//: www.chinamoney.com.cn/.

$$
\begin{aligned}
\log h_{t} & =-0.642986+0.947445 \log h_{t-1} \\
& +0.647886\left|\frac{\varepsilon_{t-1}}{\sqrt{h_{t-1}}}\right|-0.125999\left|\frac{\varepsilon_{t-2}}{\sqrt{h_{t-2}}}\right| \\
& -0.110117 \frac{\varepsilon_{t-2}}{\sqrt{h_{t-2}}}
\end{aligned}
$$

Do the ARCH effect test for the above model, and the accompanied probability of the F-statistics and LM are bigger than 0.05 . Test results indicate that the model can be accepted for there is not any Conditional Heteroscedastic.

In order to further empirically test the validity of EGARCH $(2,1)$ model, we selected 72 data of inter-bank lending rate from January to March of 2012 to make prediction. Compare the predictive value with the true value (Figure 3). From the prediction results, we could see that the predicted rates fluctuate around the real rates, and the margin between them is very small. The trend of the fitted curve is in line with the real rates curve, and the prediction error is very small which also shows the applicability of the model. Consequently, EGARCH $(2,1)$ prediction is applicable in the lending and borrowing interest rate risk management of china’s commercial banks.

\section{VaR Estimation of China's Commercial Banks}

The size of commercial bank interest rate risk is usually measured by the value at risk VaR. The so-called value at risk is the maximum possible loss given a certain confidence interval and time interval. The equation is (given confidence level $\alpha$ ):

$$
\mathrm{VaR}=W_{0}\left(E(r)-r_{\alpha}\right)
$$

In the equation, $W_{0}$ means the initial value of a financial asset or portfolio; $E(r)$ means the expected rate of

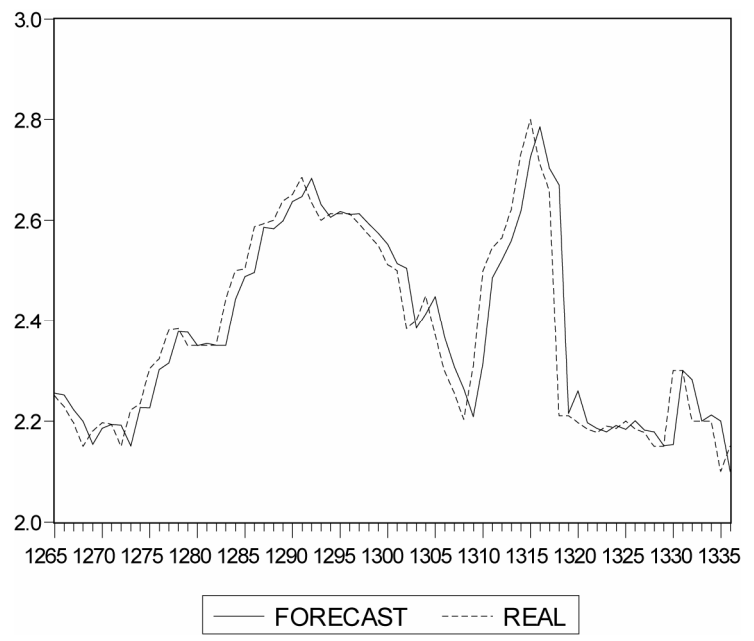

Figure 3. Predictive value compared with the actual values. 
return. Use Equation (1) to calculate the relative losses of VaR equivalent to the expected rate of return.

If the yield submits to normal distribution

" $r \sim N\left(0, \sigma_{t}^{2}\right)$ ", we could calculate sub-sites $Z_{\alpha}$, and then we could calculate " $r_{\alpha}=-Z_{\alpha} \sigma+\mu$ ”. Hence the following equation:

$$
\operatorname{VaR}=W_{0}\left(E(r)-r_{\alpha}\right)=W_{0} Z_{\alpha} \sigma
$$

However, because of the peak, thick tail and aggregation characteristics of financial time series, normal distribution will underestimate the interest rate risk.

Thus according to the previous analysis, the EGARCH $(2,1)$ conditional variance model is used to measure the VaR of interest rate market in order to solve the underestimated interest rate risk.

According to EGARCH $(2,1)$, this paper calculates the different variance in lending and borrowing market conditions (Table 5). Take conditional standard deviation into Equation (2), and dynamic VaR estimation will be got according to the net position of the main five commercial banks given the condition of $99 \%$ confidence level. And according to the daily VaR of the first quarter of five commercial banks, the dynamic VaR map (Figure 4) can be got. From the figure, the interest risk of China's commercial banks is intense.

Table 5. 2012 First 1uarter Conditions of Different Variance.

\begin{tabular}{llllll}
\hline $\mathbf{r 1}$ & 0.019198 & $\mathbf{r 2 1}$ & 0.000714 & $\mathbf{r 4 1}$ & 0.001287 \\
\hline $\mathbf{r 2}$ & 0.012258 & $\mathbf{r 2 2}$ & 0.000530 & $\mathbf{r 4 2}$ & 0.001630 \\
$\mathbf{r 3}$ & 0.008530 & $\mathbf{r 2 3}$ & 0.000906 & $\mathbf{r 4 3}$ & 0.001971 \\
$\mathbf{r 4}$ & 0.006222 & $\mathbf{r 2 4}$ & 0.000542 & $\mathbf{r 4 4}$ & 0.002027 \\
$\mathbf{r 5}$ & 0.005144 & $\mathbf{r 2 5}$ & 0.000478 & $\mathbf{r 4 5}$ & 0.002202 \\
$\mathbf{r 6}$ & 0.003876 & $\mathbf{r 2 6}$ & 0.000516 & $\mathbf{r 4 6}$ & 0.002645 \\
$\mathbf{r 7}$ & 0.002743 & $\mathbf{r 2 7}$ & 0.000403 & $\mathbf{r 4 7}$ & 0.003544 \\
$\mathbf{r 8}$ & 0.001922 & $\mathbf{r 2 8}$ & 0.000446 & $\mathbf{r 4 8}$ & 0.002305 \\
$\mathbf{r 9}$ & 0.001886 & $\mathbf{r 2 9}$ & 0.000560 & $\mathbf{r 4 9}$ & 0.001849 \\
$\mathbf{r 1 0}$ & 0.002054 & $\mathbf{r 3 0}$ & 0.000621 & $\mathbf{r 5 0}$ & 0.001699 \\
$\mathbf{r 1 1}$ & 0.001352 & $\mathbf{r 3 1}$ & 0.000515 & $\mathbf{r 5 1}$ & 0.001951 \\
$\mathbf{r 1 2}$ & 0.001609 & $\mathbf{r 3 2}$ & 0.000411 & $\mathbf{r 5 2}$ & 0.001609 \\
$\mathbf{r 1 3}$ & 0.001127 & $\mathbf{r 3 3}$ & 0.000330 & $\mathbf{r 5 3}$ & 0.001699 \\
$\mathbf{r 1 4}$ & 0.001250 & $\mathbf{r 3 4}$ & 0.000353 & $\mathbf{r 5 4}$ & 0.001644 \\
$\mathbf{r 1 5}$ & 0.000828 & $\mathbf{r 3 5}$ & 0.000384 & $\mathbf{r 5 5}$ & 0.029758 \\
$\mathbf{r 1 6}$ & 0.000821 & $\mathbf{r 3 6}$ & 0.000424 & $\mathbf{r 5 6}$ & 0.018808 \\
$\mathbf{r 1 7}$ & 0.000628 & $\mathbf{r 3 7}$ & 0.000574 & $\mathbf{r 5 7}$ & 0.013634 \\
$\mathbf{r 1 8}$ & 0.000506 & $\mathbf{r 3 8}$ & 0.000524 & $\mathbf{r 5 8}$ & 0.009183 \\
$\mathbf{r 1 9}$ & 0.000396 & $\mathbf{r 3 9}$ & 0.001812 & $\mathbf{r 5 9}$ & 0.006265 \\
$\mathbf{r 2 0}$ & 0.000900 & $\mathbf{r 4 0}$ & 0.001438 & $\mathbf{r 6 0}$ & 0.004414 \\
\hline & & &
\end{tabular}

Source: China currency (www.chinamoney.com.cn), according to the commercial bank lending position calculation of first quarter days.

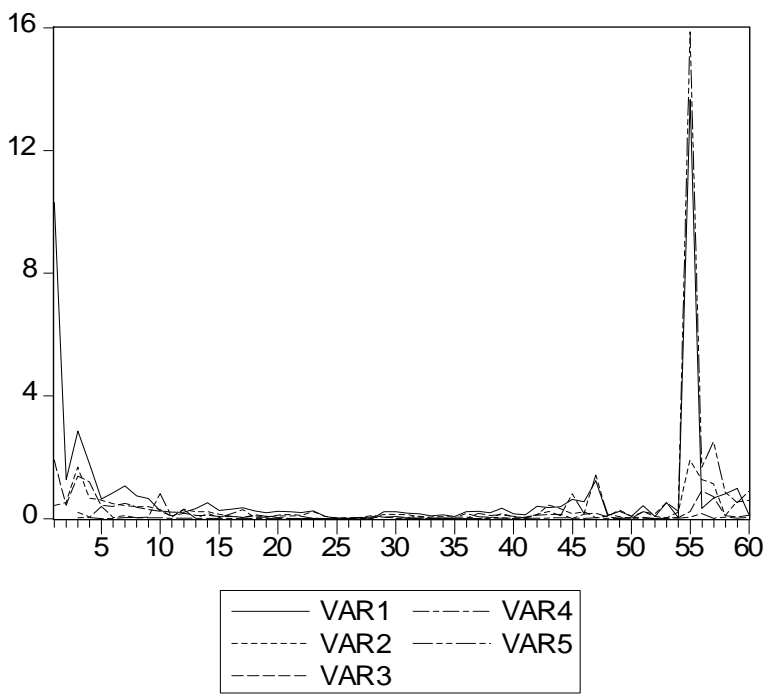

Figure 4. Dynamic VaR value of the interbank market lending position.

According to the above VaR of the first quarter of different commercial banks, by using Eviews software this paper could draw the VaR statistical analysis of China's interbank borrowing position on the 7-day dynamic for china's commercial banks (Appendix 2).

According to the statistical results of Appendix1, the standard deviation of the national commercial banks and other financial institutions is 2.168015 and 2.098728, indicating the dramatic violations in its $\mathrm{VaR}$, and the correspondingly larger risks. The VaR of city comercial banks and foreign banks is relatively small, with the standard deviation of 0.380789 and 0.353807 respectively and the performance is relatively stable. The VaR of the rural credit cooperatives are the most minimum with a standard deviation of 0.023426 , which is the most stable one in terms of the performance.

Through the analyses, it could be seen that the scale of assets and liabilities of China's rural credit cooperatives and foreign banks is relatively small, with a small amount of money lending and more borrowing money from the capital side, which means a small corresponding dynamic VaR. For national commercial banks, city commercial banks and other financial institutions, their scale of assets and liabilities is larger and more dynamic, so it has the higher risk in VaR (Appendix 3).

The results of the analysis in Appendix 1 are consistent with Appendix 2.

\section{Summary and Conclusions}

This article has proved that EGARCH $(2,1)$ model can better describe the characteristics of interest rates distribution in china's commercial interbank loan market through theoretical and empirical research. Therefore, it 
could be a useful tool for commercial banks to undergo the risk management by using VaR estimation.

Establish the model of ARCH (1), GARCH (1, 1), TGARCH $(1,1)$ and EGARCH $(2,1)$ and make successsive comparison. The AIC and SC of EGARCH $(2,1)$ is the smallest, the LL value of TGARCH $(1,1)$ is smaller than EGARCH $(2,1)$ but the fitting coefficient of EGARCH $(2,1)$ is larger than TGARCH $(1,1)$. In comprehension, EGARCH $(2,1)$ can better describe the distribution of the series of the interest rate of inter lending and borrowing market in china's commercial banks, and as a result, EGARCH $(2,1)$ could be used as the fitting model.

Use EGARCH $(2,1)$ model to calculate the interest rate market conditions heteroscedastic. Take the conditions heteroscedastic into VaR model so that the dynamic VaR estimation of night and 7-day lending position could be figured out according to the daily net trading positions, on the confidence level of 99\%. From the final results, it could be seen that the fluctuation of china's interest rate in the interbank lending and borrowing market is serious and violate, indicating china's interest rate in the interbank lending and borrowing market has been fully mar ket-oriented.

From the Bank lending and borrowing yields dynamic the $\mathrm{VaR}$ value of statistical description, it can be seen that the risk value and the standard deviation of national commercial Banks and other financial institutions is bigger, and dramatically changed. City commercial Banks and foreign banks' interest rate risk value is smaller, the performance was stable. The risk of rural credit cooperatives is the smallest and the most stable. According to the value, the risk of china's state-owned commercial Banks and other financial institutions is the largest, followed by city commercial banks and foreign banks and finally are the rural credit cooperatives the scale of assets and liabilities of China's rural credit cooperatives and foreign banks is relatively small, with a small amount of money lending and more borrowing money from the capital side, which means a small corresponding dynamic VaR. For national commercial banks, city commercial banks and other financial institutions, their scale of assets and liabilities is larger and more dynamic, so it has the higher risk in $\mathrm{VaR}$.

\section{REFERENCES}

[1] J. F. Huang, "Interest Rate Market and Commercial Bank Interest Rates,” Machinery Industry Press, Beijing, 2001.

[2] Q. Ge, "US Commercial Bank Interest Rate Risk Management," China Economic Publishing House, Beijing, 2003.

[3] G. Q. Dai, “China’s Commercial Banks Interest Rate Risk Management,” Management Research Group, Portland, 2005.

[4] Q. M. Tang and X. Gao, "An Empirical Study of the Term Structure of Interbank Lending Market in China," Statistical Research, Vol. 1, No. 5, 2001, pp. 29-31.

[5] H. F. Xu, "Asymmetric Transfer Relationship with the Efficiency of Interest Rate Policy Interest Rate," The World Economy, Vol. 1, No. 8, 2004, pp. 39-42.

[6] H. Lin and Z. L. Zheng, "The Term Structure of Interest Rates: Theory and Application,” China Financial and Economic Publishing House, Beijing, 2004.

[7] Y. Dong, "China's Short-Term Interest Rates Mean Reversion Assumption of Empirical Research,” Quantitative \& Technical Economics, Vol. 1, No. 11, 2006, pp. 151159.

[8] N. Zhang, H. X. Fei and T. Liu, "Statistics and Decision-making of China's Interbank Lending Market Interest Rate Fluctuations," Statistics and Decision, Vol. 1, No. 4, 2006, pp. 121-123.

[9] Y.-T. Cheng and Z. P. Du, "EGARCH Model in the Interbank Offered Rate Forecast," Hubei Institute for $\mathrm{Na}$ tionalities, Vol. 1, No. 6, 2007, pp. 234-237.

[10] J. Li, N. Gao and C. Cai, "Bank of China Inter-Bank Market between the Basic Characteristics of Analysis and Influencing Factors," China Accounting Review, Vol. 1, No. 1, 2007, pp. 249-266.

[11] X. Y. Liu, “Drift-Jump Process of Commercial Bank Interest Rate Risk Measurement: Theory and Experience Analysis,” Statistics and Decision, Vol. 1, No. 6, 2007, pp. 99-101.

[12] P. Jore, "Value at Risk: The New Benchmark for Controlling Market Risk,” McGraw Hill, New York, 1995.

[13] L. Chew, “Managin Derivative Risks,” John Yiley \& Soas, New York, 2002

[14] D. Duffle and J. Pan, "An Overview of Value at Risk," Journal of Derivatives, Vol. 4, No. 3, 1997, pp. 7-49. doi:10.3905/jod.1997.407971

[15] P. Jorion, "Value at Risks: Then Benchmark for Controlling Risk,” Central Press, Amman, 2001. 


\section{Appendix}

Appendix1. The inter-bank lending model comparison.

\begin{tabular}{|c|c|c|c|c|c|}
\hline Project & Coefficient & ARCH (2) & $\operatorname{GARCH}(1,1)$ & $\operatorname{TGARCH}(1,1)$ & EGARCH $(2,1)$ \\
\hline AR (2) & $\phi_{2}$ & -0.288315 & & -0.095982 & -0.117429 \\
\hline AR (3) & $\phi_{3}$ & -0.143736 & -0.088460 & -0.154973 & \\
\hline C & $\alpha_{0}$ & 0.000612 & $6.24 \mathrm{E}-05$ & 2.36E-05 & -0.643986 \\
\hline ARCH (1) & $\alpha_{1}$ & 0.000612 & 0.415604 & 0.171395 & \\
\hline ARCH (2) & $\alpha_{2}$ & 0.596140 & & & \\
\hline GARCH (1) & $\theta_{1}$ & & 0.723542 & 0.771214 & \\
\hline$($ RESID $<0) *$ ARCH (1) & $\varphi$ & & & 0.514711 & \\
\hline |RES|/SQR[GARCH] (1) & $\beta_{1}$ & & & & 0.647886 \\
\hline RES/SQR[GARCH] (1) & $\varphi_{1}$ & & & & -0.065162 \\
\hline |RES|/SQR[GARCH] (2) & $\beta_{2}$ & & & & -0.125999 \\
\hline RES/SQR[GARCH] (2) & $\varphi_{2}$ & & & & -0.110117 \\
\hline \multirow[t]{4}{*}{ EGARCH (1) } & $\theta$ & & & & 0.947445 \\
\hline & AIC & -3.634324 & -3.861316 & -3.914027 & -3.940930 \\
\hline & SC & -3.613932 & -3.845002 & -3.889557 & -3.912399 \\
\hline & LL & 2294.624 & 2436.629 & 2471.837 & 2491.757 \\
\hline R-squared & & 0.018445 & 0.006557 & 0.024882 & 0.027082 \\
\hline
\end{tabular}

Appendix 2. Commercial interbank positions dynamic VaR value (Unit: 100 Million).

\begin{tabular}{llcccc}
\hline & & Mean & Minimum & Maximum & Standard Deviation \\
\hline \multirow{2}{*}{ VAR value } & National commercial banks & 0.804891 & 0.005253 & 13.65921 & 2.168015 \\
& Foreign banks & 0.255048 & 0.000601 & 1.917143 & 0.380789 \\
& Rural credit cooperatives & 0.198768 & 0.002183 & 1.910912 & 0.353807 \\
& Other financial institutions & 0.019253 & 0.000434 & 0.166527 & 0.023426 \\
\hline
\end{tabular}

Appendix 3. Net positions of the 7-day inter-bank lending (Unit: 100 Million).

\begin{tabular}{llcccc}
\hline \multirow{2}{*}{ Lending position } & Net Position & Mean & Min & Max \\
\hline & National commercial banks & -4691.1640 & -78.18607 & -243.2900 & 197.0000 \\
& City commercial banks & 2943.8800 & 49.06467 & -9.800000 & 105.9300 \\
& Foreign banks & 1529.3140 & 25.48857 & -42.72000 & 132.7900 \\
& Rural credit cooperatives & 215.7400 & 3.595667 & -8.10000 & 25.64000 \\
& Other financial institutions & -266.7100 & -4.445167 & -228.8000 & 201.4400 \\
\hline
\end{tabular}

